\title{
Antioxidant and Anticancer Activities of Wampee (Clausena lansium (Lour.) Skeels) Peel
}

\author{
K. Nagendra Prasad, ${ }^{1}$ Jing Hao, ${ }^{1}$ Chun Yi, ${ }^{1}$ Dandan Zhang, ${ }^{1}$ Shengxiang Qiu, ${ }^{1}$ \\ Yueming Jiang, ${ }^{1}$ Mingwei Zhang, ${ }^{2}$ and Feng Chen ${ }^{3}$
}

${ }^{1}$ Key Laboratory of Plant Resources Conservation and Sustainable Utilization, South China Botanical Garden, Chinese Academy of Sciences, Guangzhou 510650, China

${ }^{2}$ Biotechnological Research Institute, Guangdong Academy of Agricultural Sciences, Guangzhou 510610, China

${ }^{3}$ Department of Food Science \& Human Nutrition, Clemson University, Clemson, SC 29634, USA

Correspondence should be addressed to Mingwei Zhang, mwzhh@163.net

Received 5 March 2009; Revised 17 May 2009; Accepted 8 June 2009

Recommended by Vickram Ramkumar

\begin{abstract}
Antioxidant activities of wampee peel extracts using five different solvents (ethanol, hexane, ethyl acetate, butanol and water) were determined by using in-vitro antioxidant models including total antioxidant capability, 1,1-diphenyl-2-picryl hydrazyl (DPPH) radical scavenging activity, reducing power, and superoxide scavenging activity. Ethyl acetate fraction (EAF) exhibited the highest antioxidant activity compared to other fractions, even higher than synthetic antioxidant butylated hydroxyl toluene (BHT). In addition, the EAF exhibited strong anticancer activities against human gastric carcinoma (SGC-7901), human hepatocellular liver carcinoma (HepG-2) and human lung adenocarcinoma (A-549) cancer cell lines, higher than cisplatin, a conventional anticancer drug. The total phenolic content of wampee fraction was positively correlated with the antioxidant activity. This is the first report on the antioxidant and anticancer activities of the wampee peel extract. Thus, wampee peel can be used potentially as a readily accessible source of natural antioxidants and a possible pharmaceutical supplement.
\end{abstract}

Copyright (C) 2009 K. Nagendra Prasad et al. This is an open access article distributed under the Creative Commons Attribution License, which permits unrestricted use, distribution, and reproduction in any medium, provided the original work is properly cited.

\section{Introduction}

In recent years, more interest has been paid to protect foods and human beings against oxidative damage caused by free radicals like hydroxyl, peroxyl, and superoxide radicals. One possible solution is to explore the potential antioxidant and anticancer properties of plant extracts or isolated products of plant origin [1]. It is well known that many polyphenolic compounds, such as phenolic acids, flavonoids, anthocyanidins, and tannins, which possess remarkable antioxidant and anticancer activities, are rich in plant materials. Some studies have shown the positive correlation of the increased dietary intake of natural antioxidants with the reduced coronary heart disease and cancer mortality, as well as with longer life expectancy $[2,3]$. Moreover, many polyphenolic compounds have shown many health-benefiting bioactive properties, such as antioxidant, anticancer, antiviral, antiinflammatory activities, and an ability to inhibit human platelet aggregation [4-6].
Wampee (Clausena lansium (Lour.) Skeels) belongs to the family Rutaceae and is originated from Southern China. The fruit which resembles grapes in appearance with acidic taste can be eaten along with the peel. The pulp can be used to prepare fruit cups, desserts, jam, or jelly. In addition, the fermented fruit can be used to prepare carbonated beverages similar to champagne, although dried wampee is a more desirable product [7]. Previous studies of bioactivities of wampee, particularly the extracts of its leaf and seed, mainly focused on the hepatoprotective [8], antiplatelet [4], hypoglycemic [9], antifungal and antiviral activities [10]. However, no systematic study has been conducted on the wampee peel even though it is used as a folk medicine in China for the treatment of stomachic, and bronchitis as well as it acts as vermifuge. For the first time, this study investigated the antioxidant and anticancer activities of the wampee peel extracts and demonstrated the potent bioactivities of the extracts suitable to be used as natural antioxidant compounds or pharmaceutical supplements. 


\section{Materials and Methods}

2.1. Plant Materials. Fresh fruit of wampee at the mature stage were obtained from an orchard in Guangzhou. Fruits were chosen for uniformity in shape and color, and washed carefully with tap water. The peels were manually separated, then dried in oven at $60^{\circ} \mathrm{C}$ for 24 hours, and finally they were grounded into powder using a blender. The moisture content of the fresh peel was determined to be $85 \%$.

2.2. Chemicals and Reagents. 1,1-Diphenyl-2-picryl hydrazyl (DPPH), nitro blue tetrazolium (NBT), methionine, riboflavin gallic acid and MTT [3-(4,5-dimethyl thiazole-2yl)2,5-diphenyl tetrazolium bromide] were purchased from Sigma Chemical Co. (St Louis, MO, USA). Trypsin and RPMI-1640 medium ( $\mathrm{pH} 7.4$ ) were obtained from GIBCO, Invitrogen, NY, USA. Fetal bovine serum was supplied by Ebioway (Guangzhou, China). Butylated hydroxyl toluene (BHT) was obtained from Sinopharm Chemical Reagent Co. (Shanghai, China). All other chemicals and solvents used in this study were of analytical grade and obtained from Tianjin Reagent Company (Tianjin, China).

2.3. Extraction. Dried powder ( $10 \mathrm{~g}$ ) of wampee peel was extracted in a rotary shaker with $200 \mathrm{~mL}$ of $50 \%$ ethanol at $30^{\circ} \mathrm{C}$ for 24 hours. The extract was then filtered, concentrated using a rotary evaporator (RE-52A, Shanghai Woshi Co. Shanghai, China), and finally lyophilized in a freezedryer (Savant, Vapornet VN 100, Labequip Ltd., Markham, Ontario, Canada) to obtain a crude ethanol fraction (CF). The extraction yield was determined to be $58.5 \pm 0.1 \%$. Partition of the CF was performed further by the method of Zhao et al. [11] with slight modifications. The freeze-dried extract was dissolved in $25 \mathrm{~mL}$ water and then partitioned sequentially by $100 \mathrm{~mL}$ of hexane, ethyl acetate, butanol and water. Hexane fraction (HF), ethyl acetate fraction (EAF), butanol fraction (BF) and water fraction (WF) were collected separately and then concentrated using a rotary evaporator to remove the solvent. The residue was dissolved in water, then freeze dried and finally stored in refrigerator until further use.

2.4. Determination of Total Phenolic Content. Total phenolic content of each fraction obtained by the above methods was determined by the method of Singleton and Rossi [12] and then expressed as microgram/gram gallic acid equivalents. In brief, a $100 \mu \mathrm{L}$-aliquot of the samples was added to $2 \mathrm{~mL}$ of $0.2 \%(\mathrm{w} / \mathrm{v}) \mathrm{Na}_{2} \mathrm{CO}_{3}$ solution. After 2 minutesutes of incubation, $100 \mu \mathrm{L}$ of $500 \mathrm{~mL} / \mathrm{L}$ Folin-Ciocalteu reagent was added and the mixture was then allowed to stand for 30 minutes at $25^{\circ} \mathrm{C}$. The absorbance was measured at 750 $\mathrm{nm}$ using a spectrophotometer (UV-2802, Unico Co. Ltd, Shanghai, China). The blank consisted of all reagents and solvents but without the sample. The total phenolic content was determined using the standard gallic acid calibration curve.

\subsection{Analyses of Antioxidant Activities}

2.5.1. Superoxide Anion Radical Scavenging Activity. Superoxide anion radical scavenging activity was measured by the method described by Duan et al. [13] with some modifications. All solutions were prepared in $0.2 \mathrm{M}$ phosphate buffer $(\mathrm{pH} 7.4)$. The wampee peel fractions at 12.5 , $25,37.5$ and $50 \mu \mathrm{g} / \mathrm{mL}$ respectively were mixed with $3 \mathrm{~mL}$ of reaction buffer solution ( $\mathrm{pH} 7.4$ ) containing $1.3 \mu \mathrm{M}$ riboflavin, $0.02 \mathrm{M}$ methionine and $5.1 \mu \mathrm{M}$ NBT. The reaction solution was illuminated by exposure to two $30 \mathrm{~W}$ fluorescent lamps for 20 minutes and then the absorbance was measured at $560 \mathrm{~nm}$ using a spectrophotometer. Butylated hydroxy toluene (BHT) was used as positive control and the reaction mixture without any sample was used as control. The superoxide anion radical scavenging activity (\%) was calculated as [1-(absorbance of sample/absorbance of control)] $\times 100$.

2.5.2. DPPH Radical Scavenging Activity. DPPH radical scavenging activities of all the fractions were determined by the method of Blois [14] with some modification. Initially, $0.2 \mathrm{~mL}$ of the fractions and BHT at a concentration of 25, 50, 75 and $100 \mu \mathrm{g} / \mathrm{mL}$ respectively, was mixed with $1 \mathrm{~mL}$ of $0.2 \mathrm{mM}$ DPPH (dissolved in methanol). The reaction mixture was incubated for 20 minutes at $28^{\circ} \mathrm{C}$ under dark condition. The control contained all reagents except the extract fraction while methanol was used as blank. The DPPH radical scavenging activity was determined by measuring the absorbance at $517 \mathrm{~nm}$ using a spectrophotometer. The DPPH radical scavenging activity (\%) of the sample was calculated as [1-(absorbance of sample/absorbance of control)] $\times 100$.

2.5.3. Total Antioxidant Activity. Total antioxidant capacities of the wampee fractions and BHT were determined by the method of Pan et al. [15]. An aliquot (0.1 mL) of these fractions was combined with $1 \mathrm{~mL}$ of reagent solution $(0.6 \mathrm{M}$ sulphuric acid, $28 \mathrm{mM}$ sodium phosphate and $4 \mathrm{mM}$ ammonium molybdate). The tubes were capped and incubated at $95^{\circ} \mathrm{C}$ for 90 minutes. After the samples were cooled to $25^{\circ} \mathrm{C}$, the absorbance was measured at $695 \mathrm{~nm}$ against blank. The blank contained $1 \mathrm{~mL}$ of reagent solution without the sample. The total antioxidant activity was expressed as the absorbance value at $695 \mathrm{~nm}$. The higher absorbance value indicates the greater antioxidant activity.

2.5.4. Reducing Power. Determination of reducing power was performed as described by Pan et al. [15] with slight modification. Various fractions $(0.1 \mathrm{~mL})$ and BHT were mixed with $2.5 \mathrm{~mL}$ of $0.2 \mathrm{M}$ phosphate buffer ( $\mathrm{pH} 6.6$ ) and $2.5 \mathrm{~mL}$ of $1 \%$ potassium ferricyanide. After the mixture was incubated at $50^{\circ} \mathrm{C}$ for 20 minutes, $2.5 \mathrm{~mL}$ of $10 \%$ trichloroacetic acid, $2.5 \mathrm{~mL}$ distilled water and $0.5 \mathrm{~mL}$ of $0.1 \%$ ferric chloride solution were added and then the absorbance was measured at $700 \mathrm{~nm}$ against a blank. The blank consists of all the reagents without the sample. Increasing absorbance of the reaction mixture indicates increasing reducing power. 


\subsection{Analysis Of Anticancer Activity}

2.6.1. Cell Line and Culture. The cell lines of HepG2 (human hepatocellular liver carcinoma), A-549, (human lung adenocarcinoma) and SGC-7901 (human gastric carcinoma) were obtained from Biomedicine Research and Development Centre of Jinan University (Guangzhou, China). The cells were cultured in a growth medium (RPMI-1640, pH 7.4), supplemented with $10 \%$ fetal bovine serum (FBS) and antibiotics, penicillin (100 units $/ \mathrm{mL}$ ), and streptomycin sulfate $(100 \mu \mathrm{g} / \mathrm{mL})$.

2.6.2. Anticancer Assay. Since EAF showed the strongest antioxidant activity in all antioxidant tests, it was chosen for anticancer assay according to the method of Zhao et al. [16]. In brief, the cells were seeded into 4 wells of a 96-well microtiter plate (Costar 3599, Corning, NY, USA) at $2 \times 10^{4}$ cells per well with $100 \mu \mathrm{L}$ RPMI-1640 growth medium and then incubated for 24 hours at $37^{\circ} \mathrm{C}$ under $5 \%$ $\mathrm{CO}_{2}$ in a humidified atmosphere (Incu-Safe, Sanyo, Japan). Later, the medium was removed while fresh growth medium containing EAF at 100, 50, 25, 12.5, 6.5 or $3.125 \mu \mathrm{g} / \mathrm{mL}$ was added. After 3 days of incubation at $37^{\circ} \mathrm{C}$ under $5 \% \mathrm{CO}_{2}$, the medium was removed while $0.1 \mathrm{mg} / \mathrm{mL}$ MTT reagent was then added. After incubation for 5 hours at $37^{\circ} \mathrm{C}$, the MTT reagent was removed before adding $100 \mu \mathrm{L}$ DMSO to each well and gently shaken. The absorbance was then determined by ELISA reader (Bio-Rad, Hercules, California, USA) at $492 \mathrm{~nm}$. Control wells received only the media without the tested samples. The conventional anticancer drug, cisplatin, was used as a positive control in this study. The inhibition of cell growth by EAF was calculated as a percent anticancer activity using the following formula: percent anticancer activity $\left(A_{c}-A_{s} / A_{c}\right) \times 100 \%$, where $A_{c}$ and $A_{s}$ referred to the absorbances of control and the sample, respectively.

2.7. Statistical Analysis. Data were expressed as means \pm standard deviations (SD) of three replicate Determinations and then analyzed by SPSS V.13 (SPSS Inc., Chicago, USA). One way analysis of variance (ANOVA) and Duncan's New Multiple-range test were used to determinutese the differences among the means. $P$ values $<.05$ were regarded as significant. Relationship between total phenolic content and antioxidant activity was determined using the Microsoft Office Excel program.

\section{Results and Discussion}

3.1. Total Phenolic Content and Extraction Yield. Table 1 shows the total phenolic content and extraction yield of each fractions of wampee peel. BF obtained the highest extraction yield $(55.16 \pm 2.5 \%)$ while HF yielded the lowest (6.86 \pm $1.16 \%)$. For the phenolics, the EAF exhibited the highest phenolic content while the HF showed the lowest. Hexane was used to extract nonpolar compounds, while ethyl acetate was used to extract medium polar flavonoids and glycosides. In contrast, butanol and water were used for extracting polar compounds like aglycones, glucosides and sugars $[17,18]$.

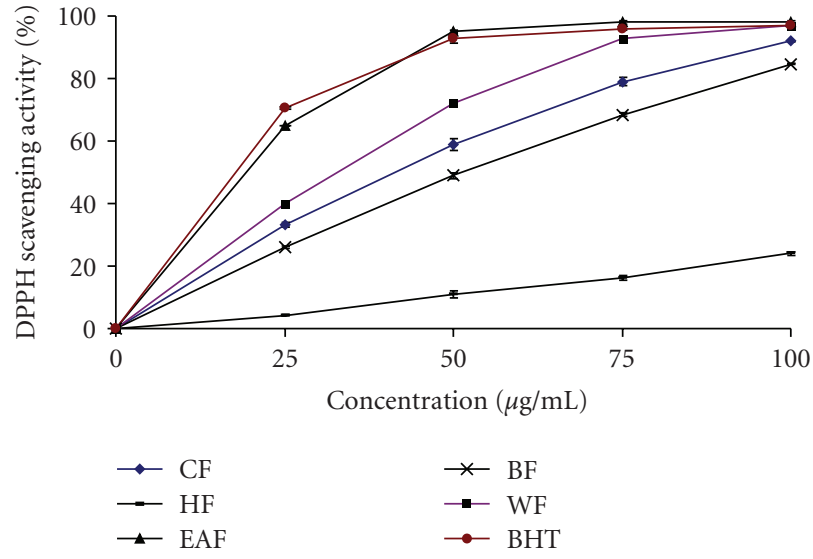

FIGURE 1: DPPH radical scavenging activity of the crude ethanol fraction (EF), hexane fraction (HF), ethyl acetate fraction (EAF), butanol fraction (BF) and water fraction (WF) from wampee peel.

According to our previous work, higher phenolic content was observed in the ethyl acetate fraction compared to the fractions of butanol and water from litchi fruit pericarp [11, 19]. Thus, EAF was used due to its relatively more efficient extraction of polyphenolic compounds from wampee peel for the further evaluation of antioxidant activity.

3.2. DPPH Radical Scavenging Activity. DPPH is a kind of stable free radical and accepts an electron or hydrogen radical to become a stable diamagnetic molecule which is widely used to investigate radical scavenging activity [14]. In the DPPH radical scavenging assay, antioxidants react with the DPPH radicals, which is a stable free radical and exists naturally in deep violet color, to turn into a yellow colored $\alpha, \alpha$-diphenyl- $\beta$-picryl hydrazine. The degree of discoloration indicates the radical-scavenging potential of the antioxidant [20].

The DPPH radical scavenging activity of all the fractions from wampee peel increased with increase in fraction concentration (Figure 1). The percent scavenging ability of EAF at $50 \mu \mathrm{g} / \mathrm{mL}$ was higher $(95 \pm 0.65)$ than that of other fractions and BHT $(93 \pm 1.6)$, with the order of EAF $>$ BHT $>\mathrm{WF}>\mathrm{CF}>\mathrm{BF}>\mathrm{HF}$. It has been found that phenolics, flavonoids and tocopherols reduce the DPPH radicals by their hydrogen donating ability $[11,13,21]$. The results obtained in this investigation reveal that all the fractions of wampee peel are free radical scavengers and able to react with the DPPH radical, which might be attributed to their electron donating ability.

3.3. Total Antioxidant Capacity. The total antioxidant capacity of wampee fractions was measured spectrophotometrically through phosphomolybdenum method, which is based on the reduction of Mo (IV) to Mo (V) by the sample analyte and the subsequent formation of green phosphate/Mo (V) compounds with a maximum absorption at $695 \mathrm{~nm}[15,22]$. A high absorbance value of the sample indicates its strong antioxidant activity. Figure 2 shows the total antioxidant activities of wampee fractions and BHT. All 
TABLE 1: Comparative analysis of extraction yield and total phenolic content of wampee peel fractions obtained using different solvents.

\begin{tabular}{lcc}
\hline Fraction & Extraction yield $(\%)^{*}$ & Total phenolic content $(\mu \mathrm{g} / \mathrm{g} \text { DW })^{*}$ \\
\hline CF & $58.5 \pm 0.09^{(\mathrm{a})}$ & $46.2 \pm 2.7^{(\mathrm{c})}$ \\
HF & $6.86 \pm 1.1^{(\mathrm{d})}$ & $7.9 \pm 0.4^{(\mathrm{e})}$ \\
EAF & $9.21 \pm 0.4^{(\mathrm{c})}$ & $330 \pm 9.9^{(\mathrm{a})}$ \\
BF & $55.16 \pm 2.5^{(\mathrm{a})}$ & $30.3 \pm 5.4^{(\mathrm{d})}$ \\
WF & $18.21 \pm 0.8^{(\mathrm{b})}$ & $54 \pm 6^{(\mathrm{b})}$ \\
\hline
\end{tabular}

* Values are mean \pm standard deviation of three replicate analyses. CF, Crude ethanol fraction; HF, Hexane fraction; EAF, Ethyl acetate fraction; BF, Butanol fraction; WF, Water fraction. For each treatment, the means in a column followed by different letters were significantly different at $P<.05$.

TABLE 2: Anticancer activities of the ethyl acetate fraction of wampee fruit pericarp and cisplatin.

\begin{tabular}{lcrr}
\hline Cell line & & \multicolumn{2}{c}{ \% Anticancer activity* } \\
& Samples & \multicolumn{2}{c}{ Concentration $(\mu \mathrm{g} / \mathrm{mL})$} \\
\hline SGC-7901 & EAF & $75.8 \pm 1.6$ & 100 \\
& Cisplatin & $34.2 \pm 2.2$ & $78.0 \pm 1.7$ \\
HepG-2 & EAF & $72.4 \pm .65$ & $63.9 \pm 2.1$ \\
& Cisplatin & $33.2 \pm 1.2$ & $77.8 \pm 0.37$ \\
A-549 & EAF & $69.2 \pm 2.1$ & $55.37 \pm 2.2$ \\
& Cisplatin & $29.97 \pm 3$ & $73.6 \pm 0.75$ \\
\hline
\end{tabular}

* Values were the means \pm standard deviations of three replicate analyses. SGC-7901 (human gastric carcinoma); HepG-2 (human hepatocellular liver carcinoma); A-549 (human lung adenocarcinoma); EAF, Ethyl acetate fraction of wampee; Cisplatin, positive control.

the fractions showed a good total antioxidant activity, which was concentration-dependent. The total antioxidant activity of EAF at $100 \mu \mathrm{g} / \mathrm{mL}$ was $2.5 \pm 0.05$, significantly being higher $(P<.05)$ than BHT with value of $1.3 \pm 0.005$. Furthermore, the total antioxidant activity of EAF at all concentrations tested was higher than BHT and other fractions. The total antioxidant capacity may be attributed to their chemical composition and phenolic acid content. Jayaprakasha et al. [17] indicated that the total antioxidant activity of citrus was due to the presence of phenolics, carotenoids, flavonoids and ascorbic acid.

3.4. Superoxide Anion Scavenging Activity. Superoxide anion, the one-electron reduced form of molecular oxygen, is a precursor of other reactive oxygen species such as hydrogen peroxide, hydroxyl radical, and singlet oxygen. Although the superoxide anion is relatively a weak oxidant, it may combine with other reactive species, such as nitric oxide, produced by macrophages, to give more reactive species $[13,20]$. In this study, the superoxide anion scavenging effects of various fractions from wampee peel were analyzed and the results are given in Figure 3. Among the wampee peel fractions tested, EAF exhibited the highest superoxide anion scavenging activity in a dose-dependent manner. All the fractions except HF exhibited excellent superoxide anion scavenging activity compared with BHT. At $50 \mu \mathrm{g} / \mathrm{mL}$, the superoxide scavenging activity of CF, HF, EAF, BF and WF were $42 \pm 3,2.5 \pm 1.8,88 \pm 2.2,47.5 \pm 5,12.9 \pm 0.2$, respectively, while BHT was $25 \pm 0.5$ only. Therefore the superoxide anion scavenging activity was in increasing order, $\mathrm{EAF}>\mathrm{BF}>\mathrm{CF}>$ $\mathrm{BHT}>\mathrm{WF}>\mathrm{HF}$. The superoxide anion scavenging activity might be due to the action of polyphenolic compounds. Also, the flavonoid molecules with polyhydroxylated substitutions on rings $A$ and $B$ and a free 3-hydroxyl substitution, conferred superoxide anion scavenging activity according to Siddhuraju et al. [23].

3.5. Reducing Power. Reducing power is also widely used in evaluating antioxidant activity of plant polyphenols. The reducing power is generally associated with the presence of reductones, which exert antioxidant action by breaking the free radical chains by donating a hydrogen atom. In this assay, the presence of reductants in the antioxidant sample causes the reduction of the $\mathrm{Fe}^{3+} /$ ferricyanide complex to the $\mathrm{Fe}^{2+} /$ ferrous form, so the reducing power of the sample can be monitored by measuring the formation of Perl's Prussian blue at $700 \mathrm{~nm}$ [24]. In the present study, the EAF from wampee peel exhibited a strong reducing power and was equal to BHT, as shown in Figure 4 . At $100 \mu \mathrm{g} / \mathrm{mL}$, the reducing power ability of EAF and BHT was $4.3 \pm 0.05$. The reducing power of wampee peel fractions is probably due to the presence of phenolic compounds which might act as electron donors.

3.6. Anticancer Activity. In this study, the anticancer activities of the EAF and cisplatin were investigated using an 3-(4,5-dimethylthiazole-2yl)-2,5-diphenyl tetrazolium bromide assay on three human cancer cell lines, SCG-7901, HepG-2 and A-549. A mitochondrial enzyme in living cells, succinate-dehydrogenase, cleaves the tetrazolium ring and converts the MTT to an insoluble purple formazan and the amount of formazan produced is directly proportional to the number of viable cells [25]. The anticancer activity 


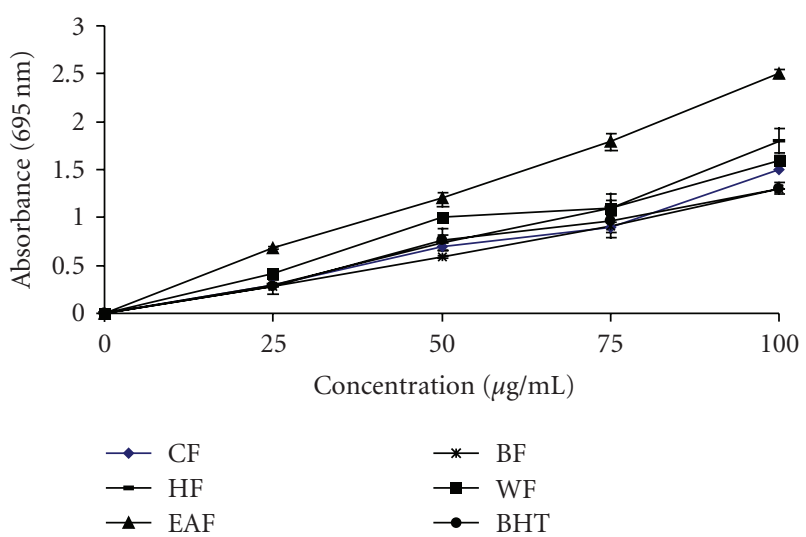

FIgURE 2: Total antioxidant capacity of the crude ethanol fraction (EF), hexane fraction (HF), ethyl acetate fraction (EAF), butanol fraction (BF) and water fraction (WF) from wampee peel. Higher absorbance value indicates higher antioxidant activity.

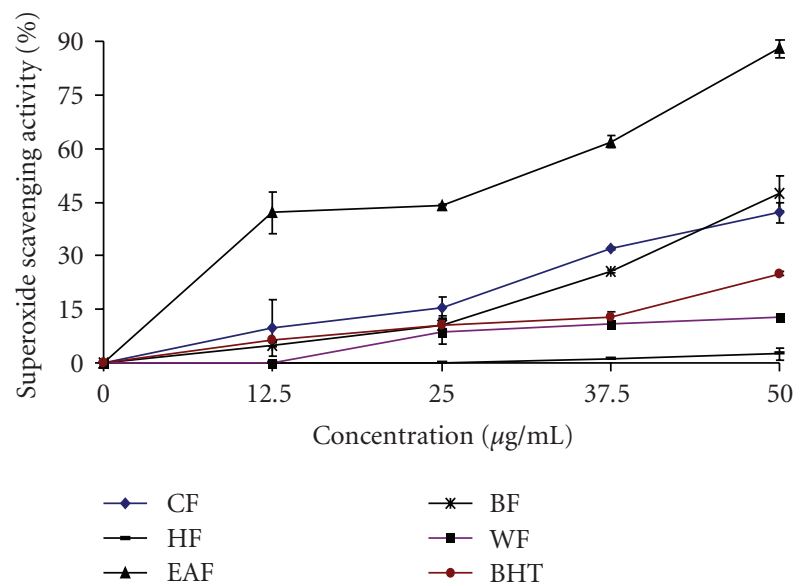

FIGURE 3: Superoxide scavenging activity of the crude ethanol fraction $(\mathrm{EF})$, hexane fraction (HF), ethyl acetate fraction (EAF), butanol fraction (BF) and water fraction (WF) from wampee peel.

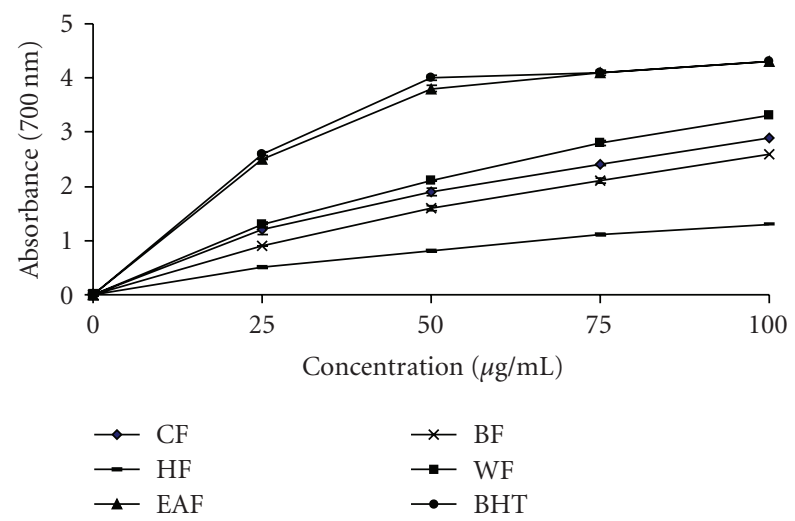

FIGURE 4: Reducing power activity of the crude ethanol fraction (EF), hexane fraction (HF), ethyl acetate fraction (EAF), butanol fraction (BF) and water fraction (WF) from wampee peel. Higher absorbance value indicates higher antioxidant activity. of EAF and cisplatin is presented in Table 2. Although the EAF inhibited the growth of cancer cells only at higher concentrations (100 and $50 \mu \mathrm{g} / \mathrm{mL}$ ), it exhibited comparable anticancer activity with cisplatin. At the concentration of $50 \mu \mathrm{g} / \mathrm{mL}$, the EAF possessed high anticancer activities against SCG-7901, HepG-2 and A-549 cancer cell lines, with the corresponding inhibitory activities of $75.8 \pm 1.6,72.4 \pm$ 0.65 and $69.2 \pm 2.1$, respectively, being significantly higher than cisplatin having values of $34.2 \pm 2.2,33.2 \pm 1.2$ and $29.97 \pm 3$, respectively.

Polyphenolic compounds might inhibit cancer cells by xenobiotic metabolizing enzymes that alter metabolic activation of potential carcinogens, while some flavonoids could also alter hormone production and inhibit aromatase to prevent the development of cancer cells [16]. The mechanism of action of anticancer activity of phenolics could be by disturbing the cellular division during mitotis at the telophase stage. It was also reported that phenolics reduced the amount of cellular protein and mitotic index, and the colony formation during cell proliferation of cancer cells [26].

Several studies exhibited a close relationship between antioxidant activities and total phenolic content $[13,15$, 21]. In this study, the antioxidant activity of wampee peel fraction was also in agreement with the total amount of phenolics, which exhibited high associations with reducing power, superoxide scavenging activity and total antioxidant capacity $\left(R^{2}=0.9159,0.9730\right.$ and 0.9228 , resp.). Thus, the content of phenolic compounds could be used as an important indicator of antioxidant capacity. Wampee peel fractions contain high levels of phenolic compounds which contain one (or more) aromatic rings bearing one or more hydroxyl groups. The more the number of hydroxyl groups in the phenolics, the greater is the antioxidant activity [27, 28]. The presence of a 4-carbonyl group of the flavonoid molecule also contributes to anticancer activity. In addition, the presence of 2,3-double bond in flavonoid molecules correlates with mitochondirial damage and cancer cell death $[29,30]$. Further investigations into the identification of these phenolic compounds present in the wampee peel are needed to better elucidate their antioxidant and anticancer activities.

\section{Conclusion}

In the present study, application of different solvents to extract antioxidant and anticancer compounds from wampee peel was investigated. The study indicated that the ethyl acetate fraction (EAF) possessed the highest phenolic content than other fractions. Also, the EAF exhibited strong antioxidant and anticancer activities, which were comparable to the commercial antioxidant BHT and the anticancer drug cisplatin. This seems that the wampee peel extract can be used as natural antioxidant and anticancer agent. Further investigation is being carried out to identify and characterize the inherent phenolic compounds responsible for the antioxidant and anticancer activities from the ethylacetate fraction of wampee peel. 


\section{Acknowledgment}

This research is supported by the CAS/SAFEA international partnership program for creative research teams.

\section{References}

[1] M. Namiki, "Antioxidants/antimutagens in food," Critical Reviews in Food Science and Nutrition, vol. 29, no. 4, pp. 273$300,1990$.

[2] B. Halliwell, "Dietary polyphenols: good, bad, or indifferent for your health?" Cardiovascular Research, vol. 73, no. 2, pp. 341-347, 2007.

[3] A. D. O. Rios, L. M. G. Antunes, and M. D. L. P. Bianchi, "Bixin and lycopene modulation of free radical generation induced by cisplatin-DNA interaction," Food Chemistry, vol. 113, no. 4, pp. 1113-1118, 2009.

[4] G.-J. Fan, B. H. Han, Y.-H. Kang, and M. K. Park, "Evaluation of inhibitory potentials of chinese medicinal plants on platelet-activating factor (PAF) receptor binding," Natural Product Sciences, vol. 7, no. 2, pp. 33-37, 2001.

[5] P. D. S. Spada, G. G. N. de Souza, G. V. Bortolini, J. A. P. Henriques, and M. Salvador, "Antioxidant, mutagenic, and antimutagenic activity of frozen fruits," Journal of Medicinal Food, vol. 11, no. 1, pp. 144-151, 2008.

[6] S. M. Mohsen and A. S. M. Ammar, "Total phenolic contents and antioxidant activity of corn tassel extracts," Food Chemistry, vol. 112, no. 3, pp. 595-598, 2009.

[7] J. Morton, "Fruits of warm climates," Miami, Fla, USA, pp. 197-198, 1987.

[8] G. T. Liu, W.-X. Li, Y.-Y. Chen, and H.-L. Wei, "Hepatoprotective action of nine constituents isolated from the leaves of Clausena lansium in mice," Drug Development Research, vol. 39, no. 2, pp. 174-178, 1996.

[9] Z. F. Shen, Q. M. Chen, H. F. Liu, and M. Z. Xie, "The hypoglycemic effect of clausenacoumarine," Acta Pharmaceutica Sinica, vol. 24, no. 5, pp. 391-392, 1989.

[10] T. B. Ng, S. K. Lam, and W. P. Fong, "A homodimeric sporamin-type trypsin inhibitor with antiproliferative, HIV reverse transcriptase-inhibitory and antifungal activities from wampee (Clausena lansium) seeds," Biological Chemistry, vol. 384, no. 2, pp. 289-293, 2003.

[11] M. Zhao, B. Yang, J. Wang, B. Li, and Y. Jiang, "Identification of the major flavonoids from pericarp tissues of lychee fruit in relation to their antioxidant activities," Food Chemistry, vol. 98, no. 3, pp. 539-544, 2006.

[12] V. L. Singleton and J. A. Rossi, "Colorimetry of total phenolics with phosphomolybdic-phosphotungstic acid reagents," American Journal of Enology and Vitriculture, vol. 16, pp. 144$158,1965$.

[13] X. Duan, G. Wu, and Y. Jiang, "Evaluation of the antioxidant properties of litchi fruit phenolics in relation to pericarp browning prevention," Molecules, vol. 12, no. 4, pp. 759-771, 2007.

[14] M. S. Blois, "Antioxidant determinations by the use of a stable free radical," Nature, vol. 181, no. 4617, pp. 1199-1200, 1958.

[15] Y. Pan, K. Wang, S. Huang, et al., "Antioxidant activity of microwave-assisted extract of longan (Dimocarpus Longan Lour.) peel," Food Chemistry, vol. 106, no. 3, pp. 1264-1270, 2008.

[16] M. Zhao, B. Yang, J. Wang, Y. Liu, L. Yu, and Y. Jiang, "Immunomodulatory and anticancer activities of flavonoids extracted from litchi (Litchi chinensis Sonn.) pericarp," International Immunopharmacology, vol. 7, no. 2, pp. 162-166, 2007.

[17] G. K. Jayaprakasha, B. Girennavar, and B. S. Patil, "Radical scavenging activities of Rio Red grapefruits and Sour orange fruit extracts in different in vitro model systems," Bioresource Technology, vol. 99, no. 10, pp. 4484-4494, 2008.

[18] K. R. Markham, Techniques of Flavonoid Identification, Academic Press, London, UK, 1982.

[19] N. K. Prasad, B. Yang, N. Ruenroengklin, M. Zhao, and Y. Jiang, "Application of ultrasonication or highpressure to assist extraction of flavonoids from litchi fruit pericarp," Journal of Food Process Engineering. In press.

[20] A. Ardestani and R. Yazdanparast, "Antioxidant and free radical scavenging potential of Achillea santolina extracts," Food Chemistry, vol. 104, no. 1, pp. 21-29, 2007.

[21] H. Li, X. Wang, Y. Li, P. Li, and H. Wang, "Polyphenolic compounds and antioxidant properties of selected China wines," Food Chemistry, vol. 112, no. 2, pp. 454-460, 2009.

[22] M. H. Abdille, R. P. Singh, G. K. Jayaprakasha, and B. S. Jena, "Antioxidant activity of the extracts from Dillenia indica fruits," Food Chemistry, vol. 90, no. 4, pp. 891-896, 2005.

[23] P. Siddhuraju, P. S. Mohan, and K. Becker, "Studies on the antioxidant activity of Indian Laburnum (Cassia fistula L.): a preliminary assessment of crude extracts from stem bark, leaves, flowers and fruit pulp," Food Chemistry, vol. 79, no. 1, pp. 61-67, 2002.

[24] N. Singh and P. S. Rajini, "Free radical scavenging activity of an aqueous extract of potato peel," Food Chemistry, vol. 85, no. 4, pp. 611-616, 2004.

[25] J.-Y. Lee, W.-I. Hwang, and S.-T. Lim, "Antioxidant and anticancer activities of organic extracts from Platycodon grandiflorum A. de Candolle roots," Journal of Ethnopharmacology, vol. 93, no. 2-3, pp. 409-415, 2004.

[26] A. Gawron and G. Kruk, "Cytotoxic effect of xanthotoxol (8hydroxypsoralen) on TCTC cells in vitro," Polish Journal of Pharmacology and Pharmacy, vol. 44, no. 1, pp. 51-57, 1992.

[27] K. N. Prasad, S. Divakar, G. R. Shivamurthy, and S. M. Aradhya, "Isolation of a free radical-scavenging antioxidant from water spinach (Ipomoea aquatica Forsk)," Journal of the Science of Food and Agriculture, vol. 85, no. 9, pp. 1461-1468, 2005.

[28] N. Rangkadilok, S. Sitthimonchai, L. Worasuttayangkurn, C. Mahidol, M. Ruchirawat, and J. Satayavivad, "Evaluation of free radical scavenging and antityrosinase activities of standardized longan fruit extract," Food and Chemical Toxicology, vol. 45, no. 2, pp. 328-336, 2007.

[29] K. Plochmann, G. Korte, E. Koutsilieri, et al., "Structureactivity relationships of flavonoid-induced cytotoxicity on human leukemia cells," Archives of Biochemistry and Biophysics, vol. 460, no. 1, pp. 1-9, 2007.

[30] L. Bravo, "Polyphenols: chemistry, dietary sources, metabolism, and nutritional significance," Nutrition Reviews, vol. 56, no. 11, pp. 317-333, 1998. 

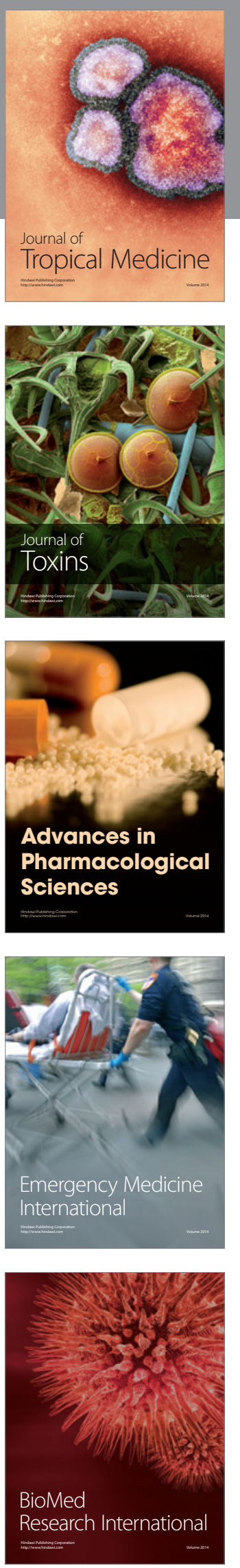
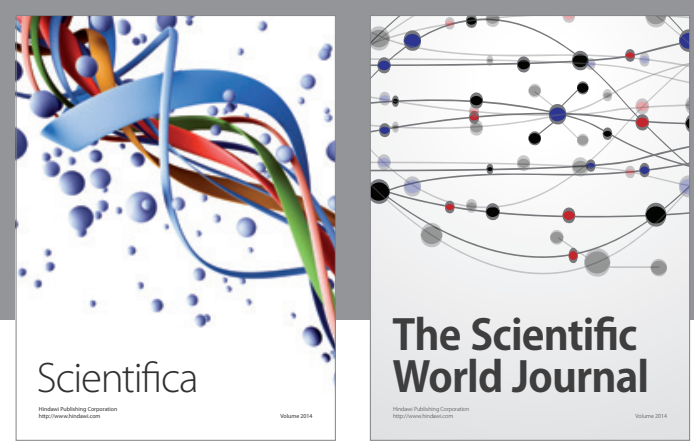

The Scientific World Journal
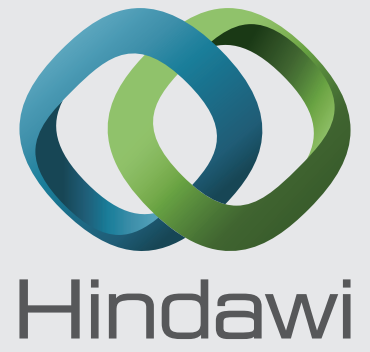

Submit your manuscripts at

http://www.hindawi.com
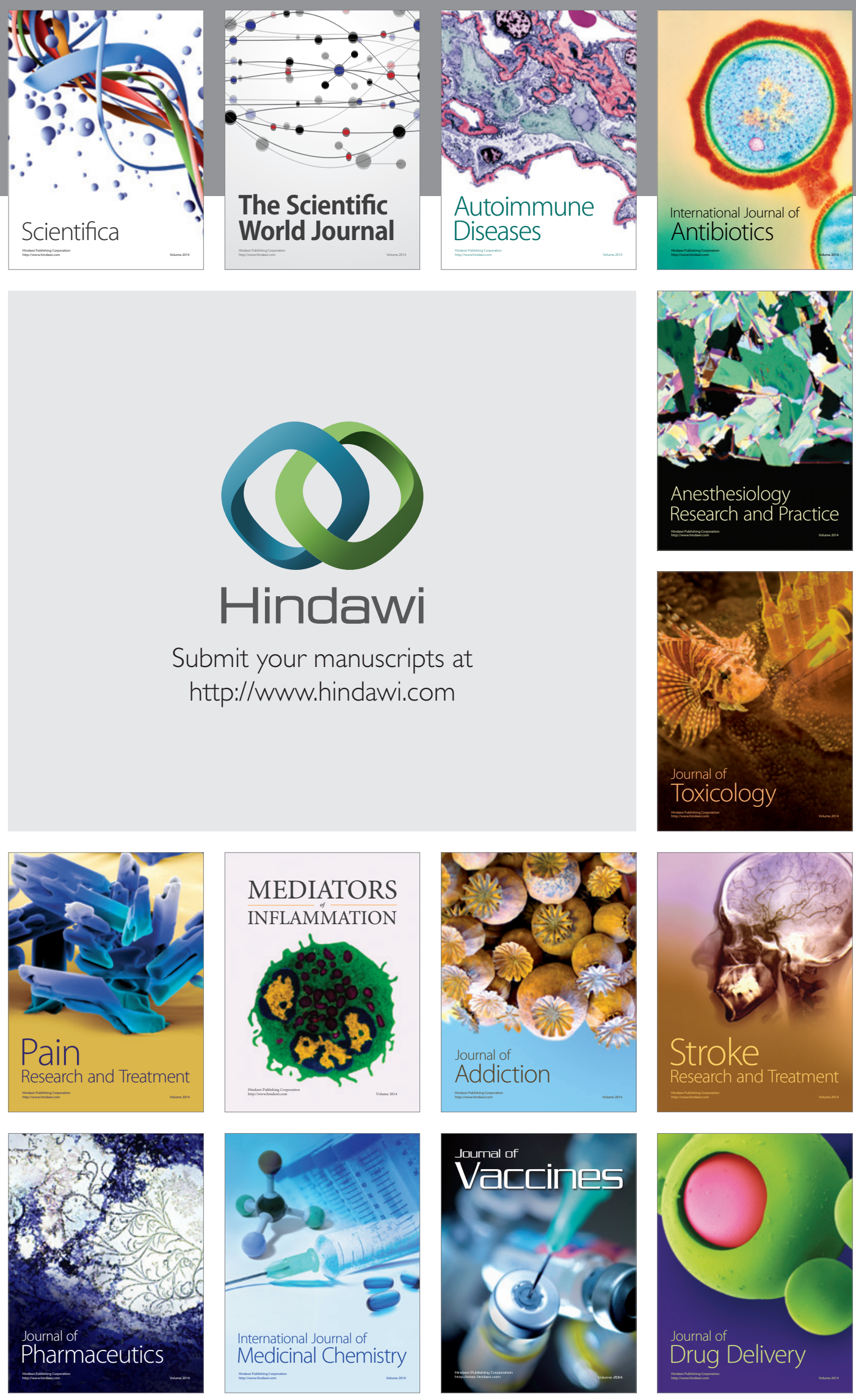